\title{
NOTES ON SUBDIRECT PRODUCTS OF SEMIGROUPS AND RECTANGULAR BANDS
}

\section{J. L. CHRISLOCK AND T. TAMURA}

1. Introduction. Let $\left\{S_{\alpha}: \alpha \in A\right\}$ be a family of semigroups. Let $p_{\alpha}$ be the natural projection from the direct product $\Pi\left\{S_{\alpha}: \alpha \in A\right\}$ onto $S_{\alpha}$. A subsemigroup $D$ of $\prod\left\{S_{\alpha}: \alpha \in A\right\}$ is called a subdirect product of $\left\{S_{\alpha}: \alpha \in A\right\}$ if $p_{\alpha}(D)=S_{\alpha}$ for all $\alpha \in A$.

A congruence $\rho$ on a semigroup $S$ is called a $\mathfrak{B}$-congruence if $S / \rho$ satisfies a property $\mathfrak{B}$. If $\mathfrak{P}$ is, for example, "semilattice," "reductivity" or "rectangular band," there exists the smallest $\mathfrak{B}$-congruence $\rho_{0}$ on $S . S / \rho_{0}$ is called the greatest $\mathfrak{B}$-homomorphic image of $S$. A semigroup $S$ is called $\mathfrak{B}$-indecomposable if any $\mathfrak{B}$-homomorphic image $Y$ of $S$ is trivial, $|Y|=1$, equivalently $\rho_{0}=S \times S$.

In this paper we will discuss how to determine all subdirect products $D$ of a semigroup $S$ and a rectangular band, and will find a necessary condition for isomorphism of subdirect products in the case where $S$ is commutative and reductive. We also will prove that the greatest semilattice-homomorphic image of $D$ is isomorphic to that of $S$.

All concepts not defined can be found in Clifford and Preston's book [1].

2. Construction of subdirect products. A rectangular band $B$ is isomorphic to the direct product $L \times R$ of a left zero semigroup $L$ and a right zero semigroup $R$ :

$$
L \times R=\{(\lambda, \rho): \lambda \in L, \rho \in R\}, \quad(\lambda, \rho)\left(\lambda^{\prime}, \rho^{\prime}\right)=\left(\lambda, \rho^{\prime}\right) .
$$

A subsemigroup $I$ of a semigroup $S$ is called a bi-ideal of $S$ if $I S I \subseteq I$. (See [1].) Let $X$ be a subset of $S$. Then $X \cup X^{2} \cup X S X$ is the smallest bi-ideal of $S$ containing $X$. If $U$ is a right ideal and $V$ a left ideal of $S$, any subset $I$ of $S$ satisfying $U V \subseteq I \subseteq U \cap V$ is a biideal of $S$. Every bi-ideal of $S$ is obtained in this manner. (See [1, p. 85].)

THEOREM 1. Let $S$ be a semigroup and $B=L \times R$ be a rectangular band. Let $\Im$ denote the set of all bi-ideals of $S$. If a mapping $\eta: B \rightarrow \Im$ satisfies

(1) $S=\cup\{\eta(a): a \in B\}$ and 1968. 
(2) $\eta(a) \cdot \eta(b) \subseteq \eta(a b)$

for all $a, b \in B$, then the set $D=\{(x, a): a \in B$ and $x \in \eta(a)\}$ is a subdirect product of $S$ and $B$. Furthermore, every subdirect product of $S$ and $B$ is obtained in this manner.

Proof. Let $\eta: B \rightarrow \Im$ satisfy (1) and (2) and define $D \subseteq S \times B$ as above. If $(s, a),(t, b) \in D$, then $(s t, a b) \in D$ by (2). Hence $D$ is a subsemigroup. Since $\eta(a) \neq \varnothing$ for all $a \in B$ and since (1) holds, $D$ is a subdirect product.

Conversely, suppose $D \subseteq S \times B$ is a subdirect product. Define a map $\eta$ from $B$ into the power set of $S$ by $\eta(a)=\{x:(x, a) \in D\}$. Since $b c b=b$ for all $b, c \in B, \eta$ obviously takes values in $\Im$. The map $\eta$ satisfies (1) since each $x \in S$ appears in some $\eta(a)$. It also satisfies (2), for if $x \in \eta(a)$ and $y \in \eta(b),(x, a)(y, b)=(x y, a b)$ or $x y \in \eta(a b)$. Finally, $D$ can be recovered from $\eta$ by noticing that $D=\{(x, a): a \in B$ and $x \in \eta(a)\}$. The proof is complete.

For the following theorem we write $(x ; \lambda, \rho)$ for the element $(x, a)$ $\in S \times B$ where $a=(\lambda, \rho)$ in $B=L \times R$.

THEOREM 2. Let $S$ be a regular semigroup and $B=L \times R$ be a rectangular band. Let $\&$ denote the set of all left ideals of $S$ and $R$ denote the set of all right ideals of $S$. Let $\phi: L \rightarrow \Re$ and $\psi: R \rightarrow \mathcal{L}$ be maps such that

(3) $U\{\phi(\lambda): \lambda \in L\}=\bigcup\{\psi(\rho): \rho \in R\}=S$.

Then the map $\eta: B \rightarrow \Im$ defined by $\eta(\lambda, \rho)=\phi(\lambda) \cap \psi(\rho)$ satisfies (1) and (2) of Theorem 1. Conversely, every map $\eta$ of Theorem 1 is obtained in this manner.

Proof. Let $\phi$ and $\psi$ satisfy (3) and define $\eta$ by $\eta(\lambda, \rho)=\phi(\lambda)$ $\cap \psi(\rho),(\lambda, \rho) \in B$. The set $\eta(\lambda, \rho)$ is obviously a bi-ideal of $S$. Also,

$$
\begin{aligned}
U\{\eta(\lambda, \rho):(\lambda, \rho) \in B\} & =\cup\{\phi(\lambda) \cap \psi(\rho):(\lambda, \rho) \in B\} \\
& =(U\{\phi(\lambda): \lambda \in L\}) \cap(U\{\psi(\rho): \rho \in R\}) \\
& =S \cap S=S,
\end{aligned}
$$

and

$$
\eta(\lambda, \rho) \cdot \eta\left(\lambda^{\prime}, \rho^{\prime}\right) \subseteq \phi(\lambda) \cdot \psi\left(\rho^{\prime}\right) \subseteq \phi(\lambda) \cap \psi\left(\rho^{\prime}\right)=\eta\left(\lambda, \rho^{\prime}\right)
$$

for all $(\lambda, \rho),\left(\lambda^{\prime}, \rho^{\prime}\right) \in B$. Thus $\eta$ satisfies (1) and (2) of Theorem 1.

Conversely, let $\eta$ satisfy (1) and (2) of Theorem 1. For $\lambda \in L$ and $\rho \in R$ define $\phi(\lambda)=\bigcup\{\eta(\lambda, \rho): \rho \in R\}$ and $\psi(\rho)=\bigcup\{\eta(\lambda, \rho): \lambda \in L\}$. By (1) and (2), 


$$
\begin{aligned}
\phi(\lambda) \cdot S & =(\bigcup\{\eta(\lambda, \rho): \rho \in R\}) \cdot\left(\bigcup\left\{\eta\left(\lambda^{\prime}, \rho^{\prime}\right):\left(\lambda^{\prime}, \rho^{\prime}\right) \in B\right\}\right) \\
& =\bigcup\left\{\eta(\lambda, \rho) \cdot \eta\left(\lambda^{\prime}, \rho^{\prime}\right): \rho \in R,\left(\lambda^{\prime}, \rho^{\prime}\right) \in B\right\} \\
& \subseteq U\left\{\eta\left(\lambda, \rho^{\prime}\right): \rho^{\prime} \in R\right\}=\phi(\lambda) .
\end{aligned}
$$

Hence $\phi: L \rightarrow R$. A similar argument shows that $\psi: R \rightarrow \mathscr{L}$. By (1), $\phi$ and $\psi$ obviously satisfy (3). We need to show that $\eta(\lambda, \rho)=\phi(\lambda) \cap \psi(\rho)$. Clearly $\eta(\lambda, \rho) \subseteq \phi(\lambda) \cap \psi(\rho)$. So suppose $x \in \phi(\lambda) \cap \psi(\rho)$. Then $x \in \eta\left(\lambda, \rho^{\prime}\right)$ and $x \in \eta\left(\lambda^{\prime}, \rho\right)$ for some $\left(\lambda^{\prime}, \rho^{\prime}\right) \in B$. But there exists $a \in S$ such that $x a x=x$. Hence, if $a \in \eta\left(\lambda^{\prime \prime}, \rho^{\prime \prime}\right)$,

$$
x=x a x \in \eta\left(\lambda, \rho^{\prime}\right) \eta\left(\lambda^{\prime \prime}, \rho^{\prime \prime}\right) \eta\left(\lambda^{\prime}, \rho\right) \subseteq \eta(\lambda, \rho)
$$

by (2). This completes the proof.

3. Further results. Let $S_{i}$ be a semigroup, $B_{i}$ a rectangular band, and $D_{i}$ a subdirect product of $S_{i}$ and $B_{i}(i=1,2)$. If $D_{1} \cong D_{2}$, it is not necessarily true that $S_{1} \cong S_{2}$ and $B_{1} \cong B_{2}$. We can, however, conclude this if the semigroups $S_{1}$ and $S_{2}$ are commutative and reductive.

A semigroup $S$ is called reductive if $a x b=a y b$ for all $a, b \in S$ implies $x=y$. If $S$ is commutative, this condition is equivalent to: $a x=a y$ for all $a \in S$ implies $x=y$.

THEOREM 3. Let $D_{i}$ be a subdirect product of a rectangular band $B_{i}$ and a semigroup $S_{i}$ which is commutative and reductive $(i=1,2)$. If $D_{1} \cong D_{2}$, then $B_{1} \cong B_{2}$ and $S_{1} \cong S_{2}$.

Proof. In this proof, $p_{S_{i}}$ and $p_{B_{i}}$ denote the natural projections of $S_{i} \times B_{i}$ onto $S_{i}$ and $B_{i}$ respectively $(i=1,2)$. First we will prove that for $x, y \in D_{i}$

(4) $p_{B_{i}}(x)=p_{B_{i}}(y)$ if and only if $x y=y x$,

(5) $p_{s_{i}}(x)=p_{s_{i}}(y)$ if and only if $a x b=a y b$ for all $a, b \in D_{i}$.

If $x y=y x$, then $p_{B_{i}}(x) p_{B_{i}}(y)=p_{B_{i}}(x y)=p_{B_{i}}(y x)=p_{B_{i}}(y) p_{B_{i}}(x)$ which implies $p_{B_{i}}(x)=p_{B_{i}}(y)$ since $B_{i}$ is a rectangular band. The converse is immediate because $S$ is commutative. This proves (4). To prove (5), suppose $a x b=a y b$ for all $a, b \in D_{i}$. Then we have

$$
p_{s_{i}}(a) p_{s_{i}}(x) p_{s_{i}}(b)=p_{s_{i}}(a) p_{s_{i}}(y) p_{s_{i}}(b) \quad \text { for all } a, b \in D_{i} \text {. }
$$

This implies $p_{S_{i}}(x)=p_{S_{i}}(y)$ since $p_{s_{i}}$ is onto and $S$ is reductive. The converse is obvious. Thus we have (5).

If we define $x \beta_{i} y$ by $p_{B_{i}}(x)=p_{B_{i}}(y)$ and $x \gamma_{i} y$ by $p_{S_{i}}(x)=p_{S_{i}}(y)$, then (4) and (5) show that $\beta_{i}$ and $\gamma_{i}$ are not in terms of the projections $p_{B_{i}}$ and $p_{S_{i}}$ respectively. It is easy to see that $\beta_{i}$ is the smallest rectangular-band congruence on $D_{i}$ and $\gamma_{i}$ is the smallest reductive congruence 
on $D_{i}$, and $D_{i} / \beta_{i} \cong B_{i}, D_{i} / \gamma_{i} \cong S_{i}$. Since $D_{1} \cong D_{2}$, we have $B_{1} \cong B_{2}$ and $S_{1} \cong S_{2}$.

REMARK. In (4) we have used only commutativity of $S$. Accordingly if $S$ is commutative and if $D_{1} \cong D_{2}$, then $B_{1} \cong B_{2}$.

THeOREM 4. Let $S$ be a semigroup and $B$ a rectangular band. The greatest semilattice-homomorphic image of a subdirect product of $S$ and $B$ is isomorphic to that of $S$.

Proof. Let $D$ be a subdirect product of $S$ and $B$. Every element of $D$ is denoted by $(t, a), t \in S, a \in B$. Let $\sigma$ be the congruence on $D$ defined by

$$
(t, a) \sigma(s, b) \quad \text { if and only if } t=s .
$$

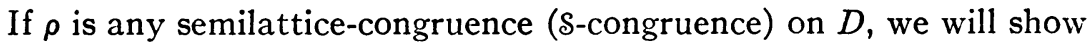
that $\sigma \subseteq \rho$. To do this we will use the fact that $x y x=x$ for elements of a rectangular band. Let $(t, a),(t, b) \in D$.

$$
\begin{aligned}
(t, a) \rho\left(t^{4}, a b^{2} a\right) & =(t, a)(t, b)(t, b)(t, a) \\
\rho(t, b)(t, a)(t, a)(t, b) & =\left(t^{4}, b a^{2} b\right) \rho(t, b) .
\end{aligned}
$$

Hence $\sigma \subseteq \rho$ for all $\delta$-congruences $\rho$. Each $\rho$ induces an $\delta$-congruence $\bar{\rho}$ on $D / \sigma$; conversely, an $\delta$-congruence $\bar{\rho}$ on $S \cong D / \sigma$ induces an $\delta$ congruence $\rho$ on $D$ with $\sigma \subseteq \rho$. Therefore the smallest $S$-congruence $\rho_{0}$ on $D$ corresponds to the smallest $S$-congruence $\bar{\rho}_{0}$ on $S$ in the above sense. Consequently the greatest $S$-homomorphic image of $D$ is isomorphic to that of $S$.

CoRollary. If $S$ is s-indecomposable then any subdirect product of $S$ and $B$ is $S$-indecomposable. Conversely if one subdirect product of $S$ and $B$ is $\mathrm{S}$-indecomposable, then $S$ is also.

\section{REFERENCE}

1. A. H. Clifford and G. B. Preston, The algebraic theory of semigroups, Vol. I, Math. Surveys No. 7, Amer. Math. Soc., Providence, R. I., 1961.

University of California, Santa Cruz and University of California, Davis 